Supporting Information for

\title{
Circularly Polarized Luminescence from Achiral Single Crystals of Hybrid Manganese Halides
}

Jian Zhao ${ }^{\dagger,+}$, Tongjin Zhang ${ }^{\ddagger},+$ Xi-Yan Dong ${ }^{\dagger \S}$, Meng-En Sun ${ }^{\dagger}$, Chong Zhang ${ }^{\dagger}$, Xinlei $\mathrm{Li}^{\dagger}$, Yong Sheng Zhao ${ }^{\ddagger *}$, Shuang-Quan Zang ${ }^{\dagger *}$

${ }^{\dagger}$ College of Chemistry and Molecular Engineering, Zhengzhou University, Zhengzhou 450001, China

${ }^{\ddagger}$ CAS Key Laboratory of Photochemistry, Institute of Chemistry, Chinese Academy of Sciences,Beijing 100190, China

${ }^{\S}$ College of Chemistry and Chemical Engineering, Henan Polytechnic University, Jiaozuo 454000, China 


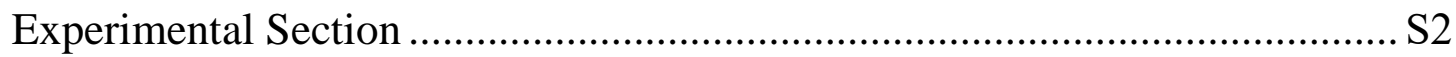

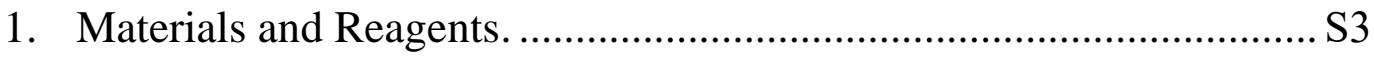

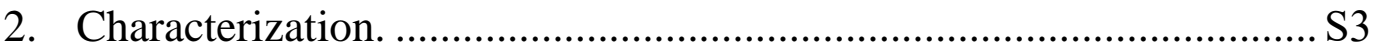

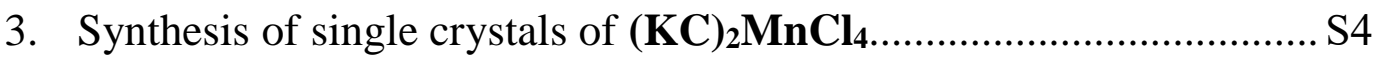

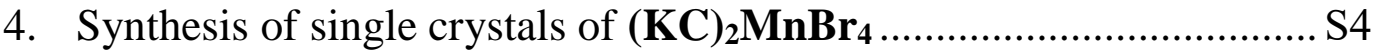

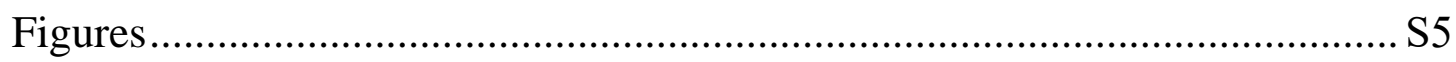

Figure S1. The images of reaction progress record.................................... S5

Figure S2. The layer constructed by helixes through $\mathrm{K}^{+}-\pi$ interactions .... S5

Figure S3. Powder X-ray diffraction (PXRD) patterns .............................. S6

Figure S4. Thermogravimetric (TG) analysis ............................................ S6

Figure S5. Solid-state UV-vis diffuse reflectance spectra …………......... S6

Figure S6. Characterization of basic optical properties of $(\mathbf{K C})_{2} \mathbf{M n B r}_{4}$.. S7

Figure S7. Experimental setup for the CPL and optical waveguide .......... S7

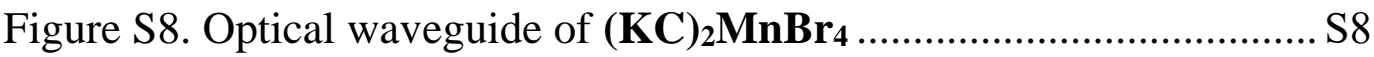

Figure S9. CPL of $(\mathbf{K C})_{2} \mathbf{M n C l}_{4}$ single crystal ...................................... S8

Figure S10. CPL of $(\mathbf{K C})_{2} \mathbf{M n B r}_{4}$ and $(\mathbf{K C})_{2} \mathbf{M n C l}_{4}$ powder sample ........ S9 Tables S10-S12

Table S1. Crystal data and structure refinement ...................................... S10

Table S2. Selected bond distances and bond angles for $(\mathbf{K C})_{2} \mathbf{M n C l}_{4} \ldots . \mathrm{S} 11$

Table S3. Selected bond distances and bond angles for $(\mathbf{K C})_{2} \mathbf{M n B r}_{4} \ldots . \mathrm{S} 11$

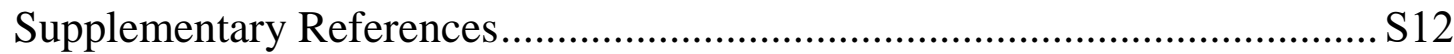




\section{A. Experimental Section}

\section{Materials and Reagents.}

Analytically pure $\mathrm{MnCl}_{2} \cdot 4 \mathrm{H}_{2} \mathrm{O}, \mathrm{MnBr}_{2} \cdot 4 \mathrm{H}_{2} \mathrm{O}, \mathrm{KCl}, \mathrm{KBr}$, dibenzo-18-crown-6, Ethanol (EtOH) and Methylene chloride (DCM) were commercial available and used without future purification.

\section{Characterization.}

Single-crystal X-ray diffraction data were collected on a Rigaku XtaLAB Pro diffractometer with $\mathrm{Cu}-\mathrm{K} \alpha$ radiation $(\lambda=1.5418 \AA)$ at $170 \mathrm{~K}$ for $(\mathbf{K C})_{2} \mathbf{M n C l}_{4}$ and $(\mathbf{K C})_{2} \mathbf{M n B r}_{4}$. Data collection and reduction were performed using the program CrysAlisPro. ${ }^{1}$ The crystal structures were solved by direct methods $(S H E L X S)^{2}$ and refined by full-matrix least squares on $\mathrm{F} 2$ using $O L E X^{2},{ }^{3}$ with anisotropic thermal displacement parameters employed for all nonhydrogen atoms, which utilizes the SHELXL-2015 module. ${ }^{4}$ X-ray powder diffraction (PXRD) patterns of the samples were recorded on a Rigaku MiniFlex600 diffractometer. Simulated powder patterns were calculated by Mercury software using the crystallographic information file (CIF) from a single-crystal X-ray experiment. Thermogravimetry analyses (TGA) were performed on a TA Q50 system from room temperature to $400^{\circ} \mathrm{C}$ under a $\mathrm{N}_{2}$ atmosphere (flow rate $=60 \mathrm{~mL} / \mathrm{min}$ ) at a heating rate of $10^{\circ} \mathrm{C} / \mathrm{min}$. Solid-state UV-vis diffuse reflectance spectra were recorded using a Hitachi UH4150 UV-vis spectrophotometer. Room temperature steady-state spectroscopy, time-resolved photoluminescence (PL) and photoluminescence quantum yields measurements were carried out on a HORIBA FluoroLog-3 fluorescence spectrometer. Luminescence microscopy images were recorded on an Olympus BX53 microscope using a xenon lamp as excitation source. The single-crystal microrods were locally excited by a focused laser beam filtered with a bandpass filter (330-380 nm) using an objective lens $(20 \times$ N.A. $=0.50)$. The spatially resolved spectra were measured with a grating spectrometer (Acton SP-2358) connected to a an thermal-electrically cooled CCD EMCCD (Princeton Instrument ProEM 1600B). Polarization profiles of the emission 
intensity were obtained by setting a quarter-waveplate (THORLABS AQWP05M-600) and a linear polarizer (THORLABS LPVISC050) in front of the spectrometer. Circularly polarized luminescence (CPL) spectra of powder samples were measured on a JASCO CPL-300.

\section{Synthesis of single crystals of $(\mathrm{KC})_{2} \mathrm{MnCl}_{4}$}

$\mathrm{MnCl}_{2} \cdot 4 \mathrm{H}_{2} \mathrm{O}(0.4007 \mathrm{~g}, 2.025 \mathrm{mmol}), \mathrm{KCl}(0.0997 \mathrm{~g}, 1.337 \mathrm{mmol})$, dibenzo-18crown-6 (0.4812 g, $1.335 \mathrm{mmol})$ were dissolved in $10 \mathrm{ml} \mathrm{EtOH}$ and $16 \mathrm{ml} \mathrm{DCM}$ at room temperature. The light green needle-like crystal can be obtained simply and quickly by room temperature solvent evaporation. Yield: $60.5 \%$ based on dibenzo-18crown-6.

\section{Synthesis of single crystals of $(\mathrm{KC})_{2} \mathrm{MnBr}_{4}$}

$\mathrm{MnBr}_{2} \cdot 4 \mathrm{H}_{2} \mathrm{O}(0.4007 \mathrm{~g}, 1.397 \mathrm{mmol}), \mathrm{KBr}(0.1393 \mathrm{~g}, 1.171 \mathrm{mmol})$, dibenzo-18crown-6 (0.4810 g, $1.335 \mathrm{mmol})$ were dissolved in $8 \mathrm{ml} \mathrm{EtOH}$ and $16 \mathrm{ml} \mathrm{DCM}$ at room temperature. The light green needle-like crystal can be obtained simply and quickly by room temperature solvent evaporation. Yield: $28.4 \%$ based on $\mathrm{KBr}$. 


\section{B. Supporting Figures}
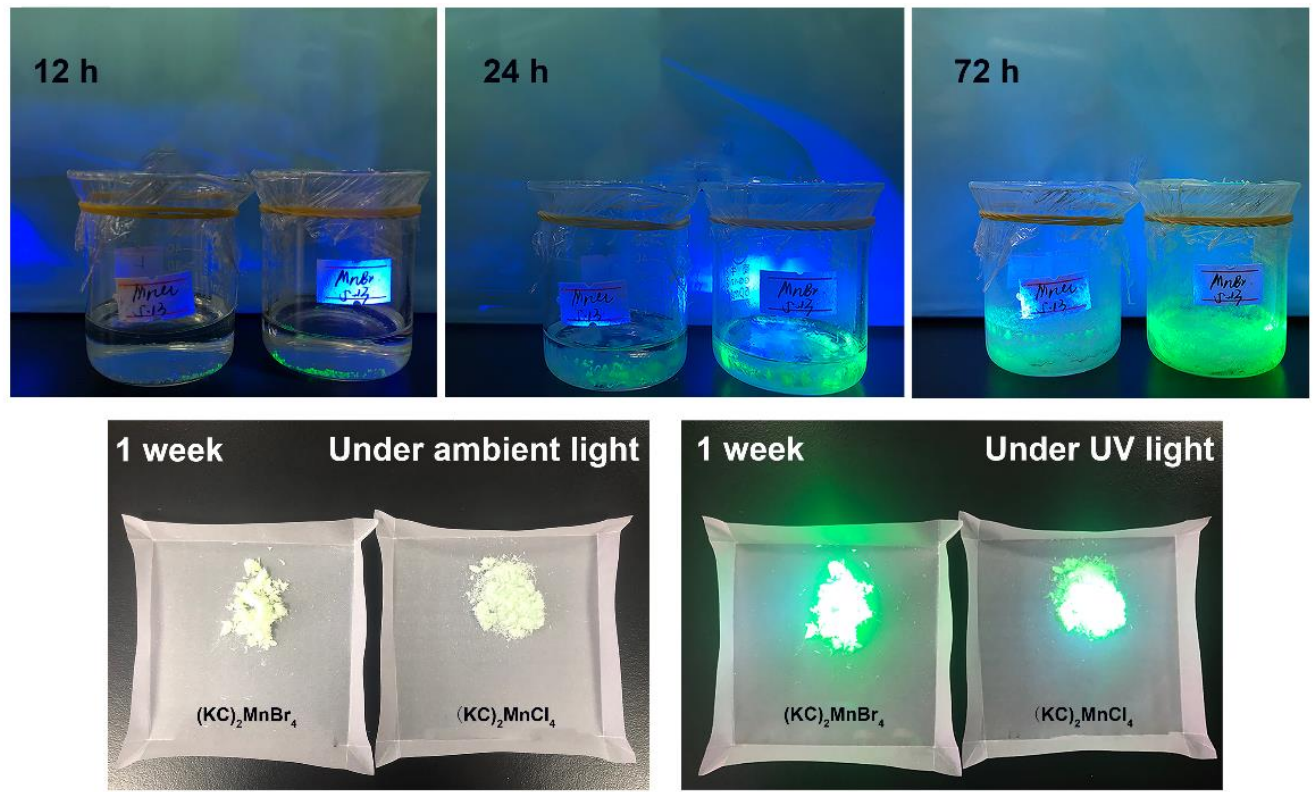

Figure S1. Images of the reaction progress recorded at 12 h, 24 h, 72 h, and 1 week. The crystals can be quickly obtained by facile ambient temperature solvent volatilization method after $12 \mathrm{~h}$ (the UV light is from a portable ultraviolet lamp).

b<smiles>[Te]=[Te]</smiles>

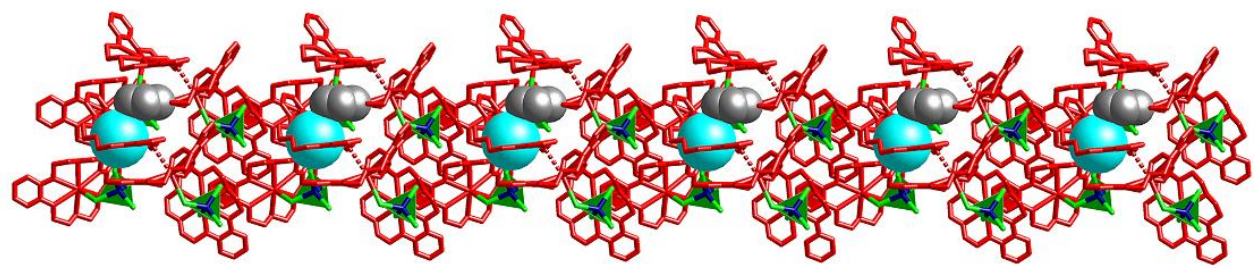

$b-t$

Right-handed helixes

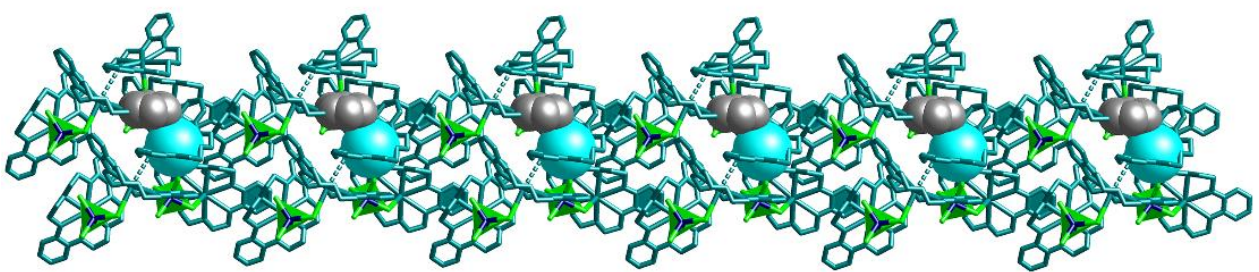

Left-handed helixes

Figure S2. The right-handed helixes and left-handed helixes can be arranged in parallel to form a two-dimensional layer through $\mathrm{K}^{+}-\pi$ interactions between helixes. (spacefilling pattern of the $\mathrm{K}^{+}-\pi$ interactions) 
(a)

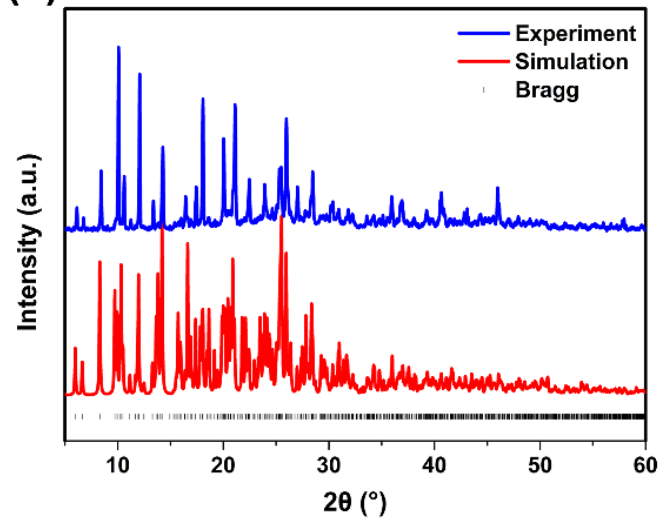

(b)

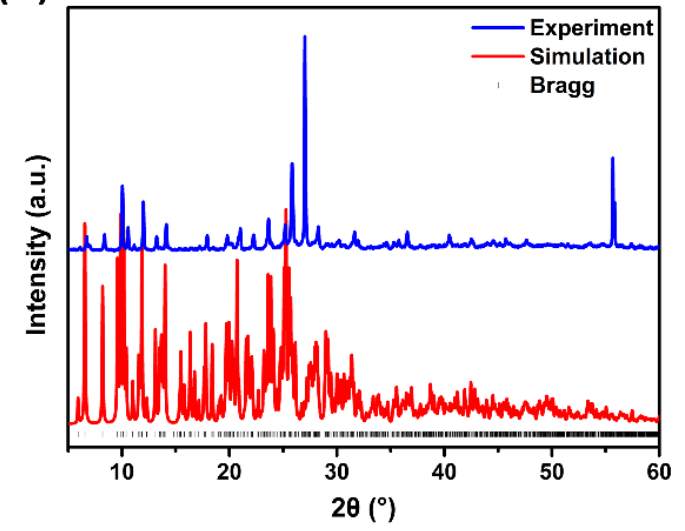

Figure S3. Experimental PXRD patterns (blue line) and patterns simulated from singlecrystal data (red line) of $\left(\mathbf{K C}_{2} \mathbf{M n C l}_{4}\right.$ (a) and $(\mathbf{K C})_{2} \mathbf{M n B r}_{4}$ (b).

(a)

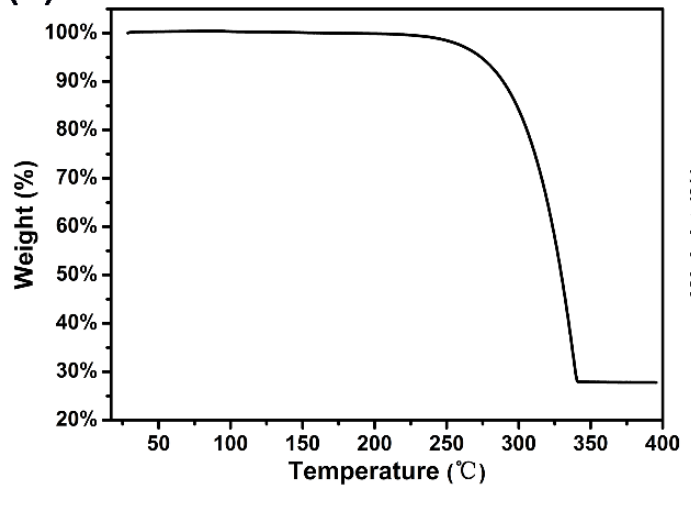

(b)

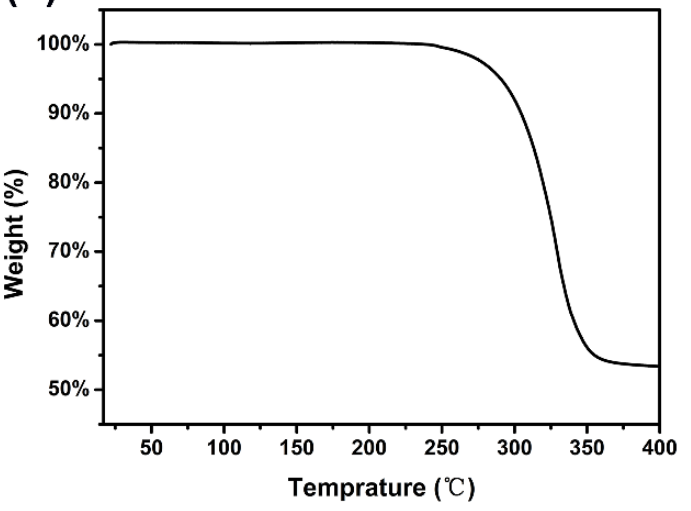

Figure S4. Thermogravimetric (TG) plots of ( $\mathrm{KC}_{2} \mathbf{M n C l}_{4}$ (a) and (KC) ${ }_{2} \mathbf{M n B r}_{4}$ (b).

(a)

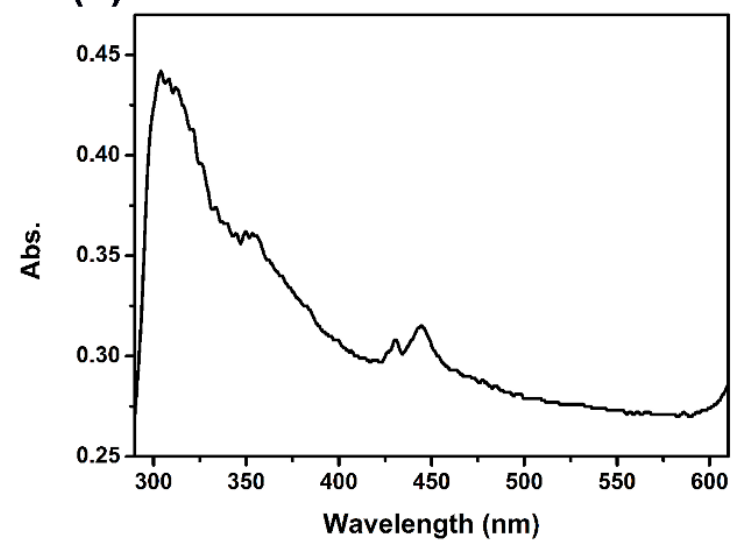

(b)

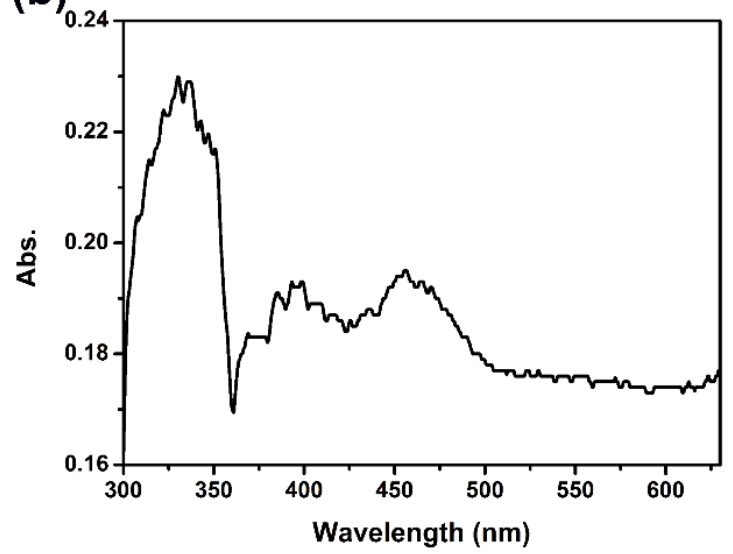

Figure S5. Solid-state UV-vis diffuse reflectance spectra of $(\mathbf{K C})_{2} \mathbf{M n C l}_{4}$ (a) and $(\mathbf{K C})_{2} \mathbf{M n B r}_{4}$ (b) powder samples. 

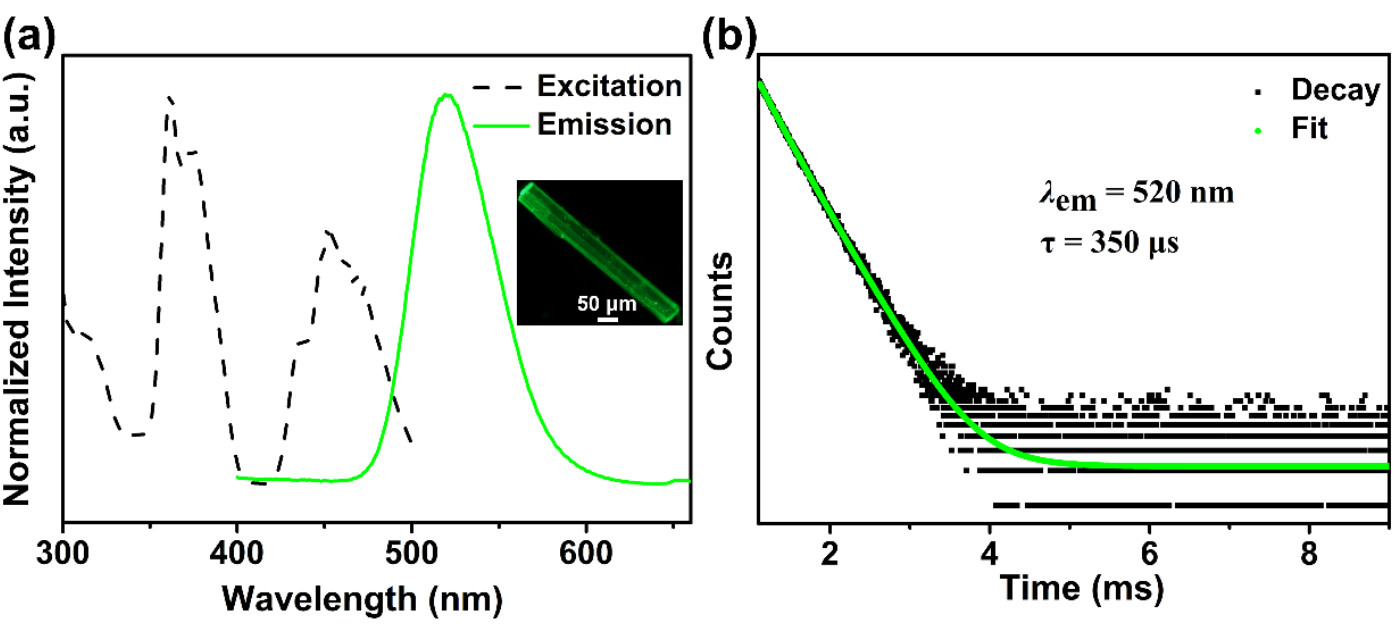

Figure S6. (a) Excitation spectra (monitored at $518 \mathrm{~nm}$ ) and emission spectra of $(\mathbf{K C})_{2} \mathbf{M n B r}_{4}$ (excited at $361 \mathrm{~nm}$ ). Inset: PL microscopy image under unfocused UV light (excited at ca. $365 \mathrm{~nm}$ ). (b) PL decay curve of (KC) $2 \mathbf{M n B r} 4$ monitored at $520 \mathrm{~nm}$.

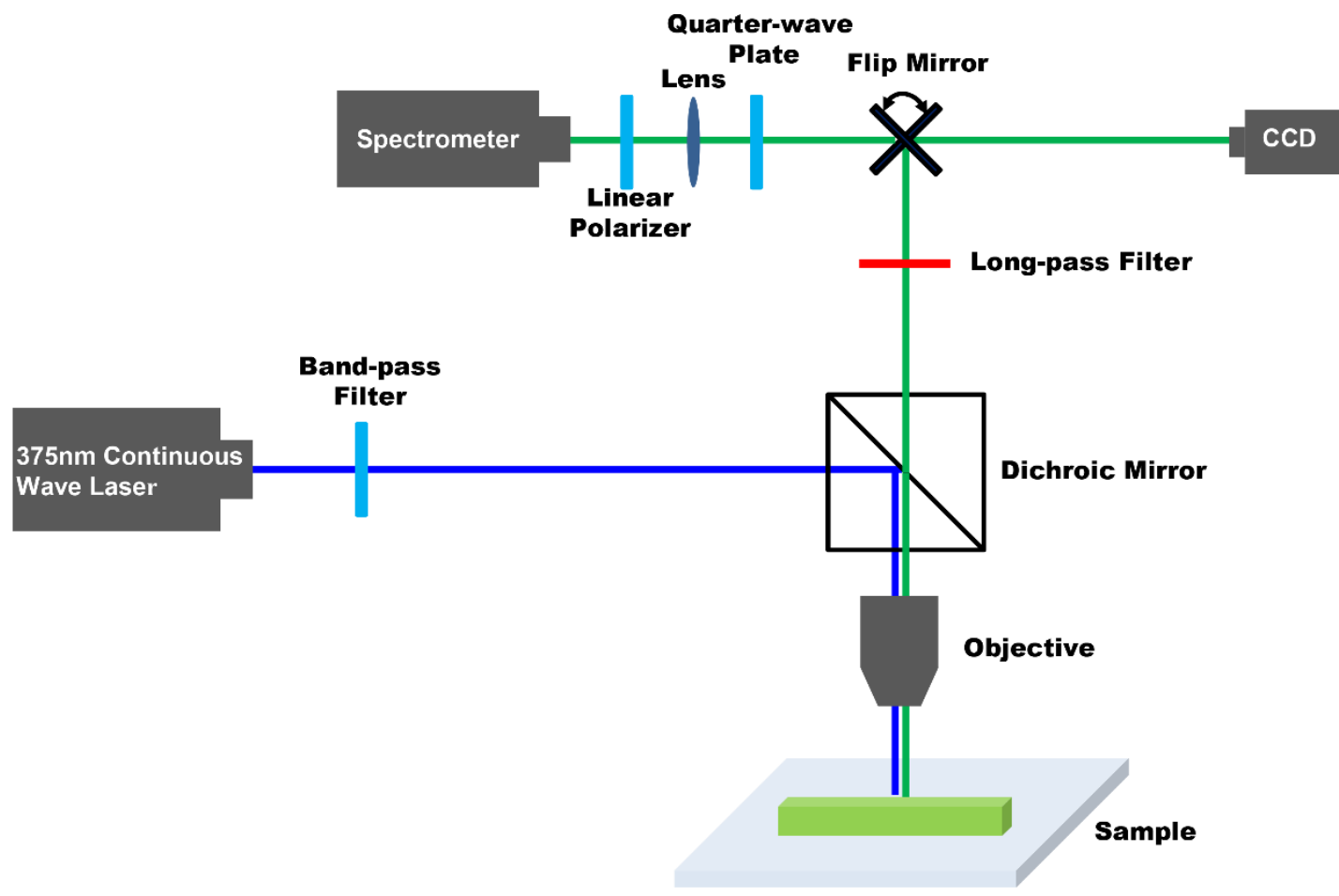

Figure S7. Schematic illustration of the experimental setup for the circularly polarized luminescence and optical waveguide (after removing the linear polarizer and $\lambda / 4$ waveplate) characterization of a single crystal. 
(a)

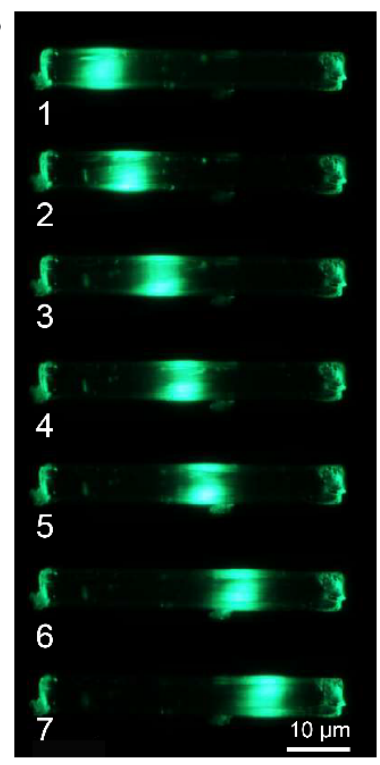

(b)
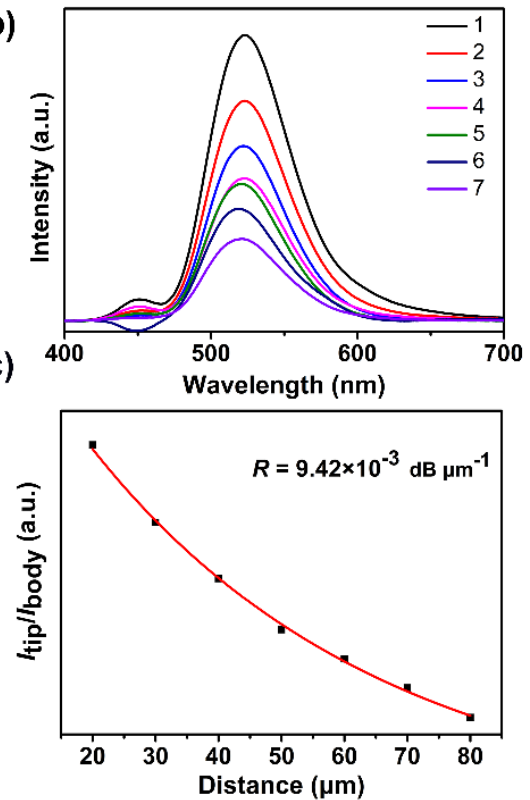

Figure S8. (a) PL images of a single-crystal 1D microrod of (KC) ${ }_{2} \mathbf{M n B r} 4$ excited at different positions (images 1-7) by focused UV light. (b) Corresponding spatially resolved PL spectra from the tip of the microrod for different distances between the excitation spot and tip of the rod. (c) Ratio of the intensity $I_{\text {tip }} / I_{\text {body }}$ versus the distance $D$. The curve was fitted by an exponential decay function, $I_{\text {tip }} / I_{\text {body }}=A \exp (-R D)$.

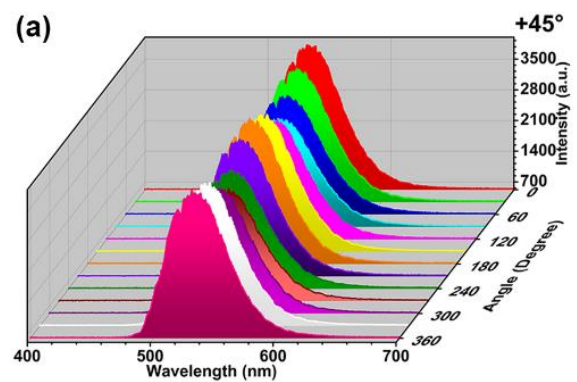

(c)
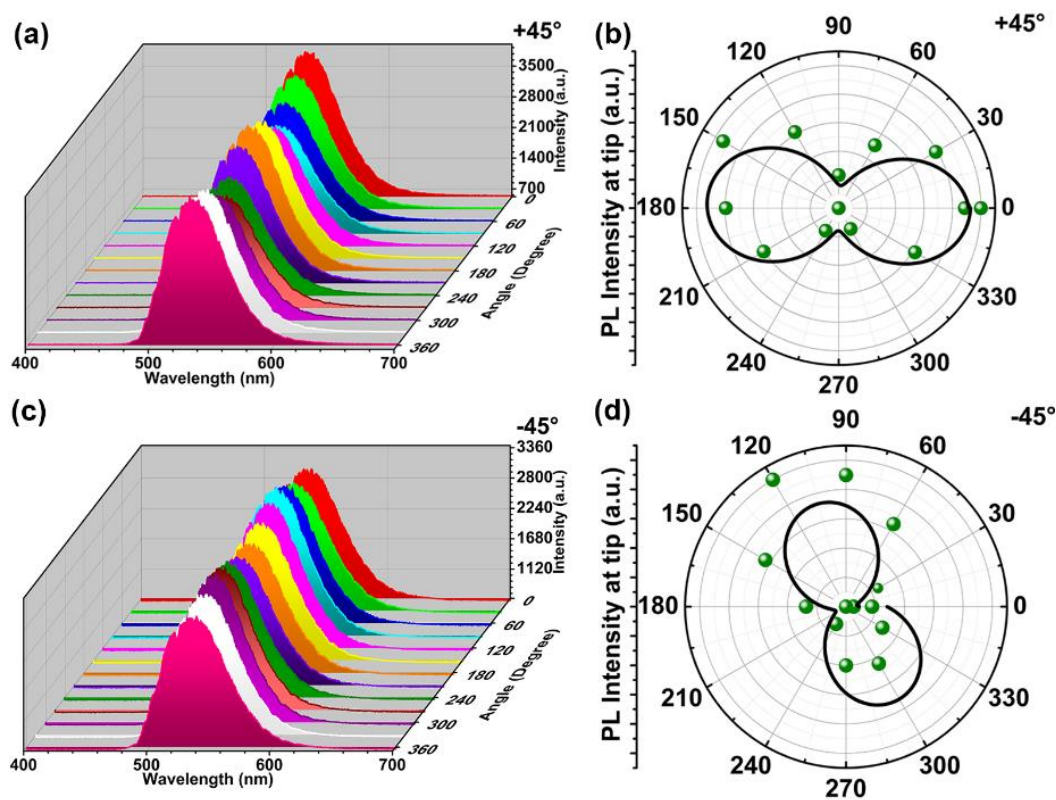

Figure S9. Polarized PL spectra of a single-crystal (KC) ${ }_{2} \mathbf{M n C l}_{4}$ microrod at various angles (ca. $0^{\circ}-360^{\circ}$ ), angle of $\lambda / 4$ waveplate $=+45^{\circ}$ (a) and $-45^{\circ}$ (c). PL intensity of the $(\mathbf{K C})_{2} \mathbf{M n C l}_{4}$ microrod as a function of the polarizer rotation angle, angle of $\lambda / 4$ waveplate $=+45^{\circ}(\mathrm{b})$ and $-45^{\circ}(\mathrm{d})$. 

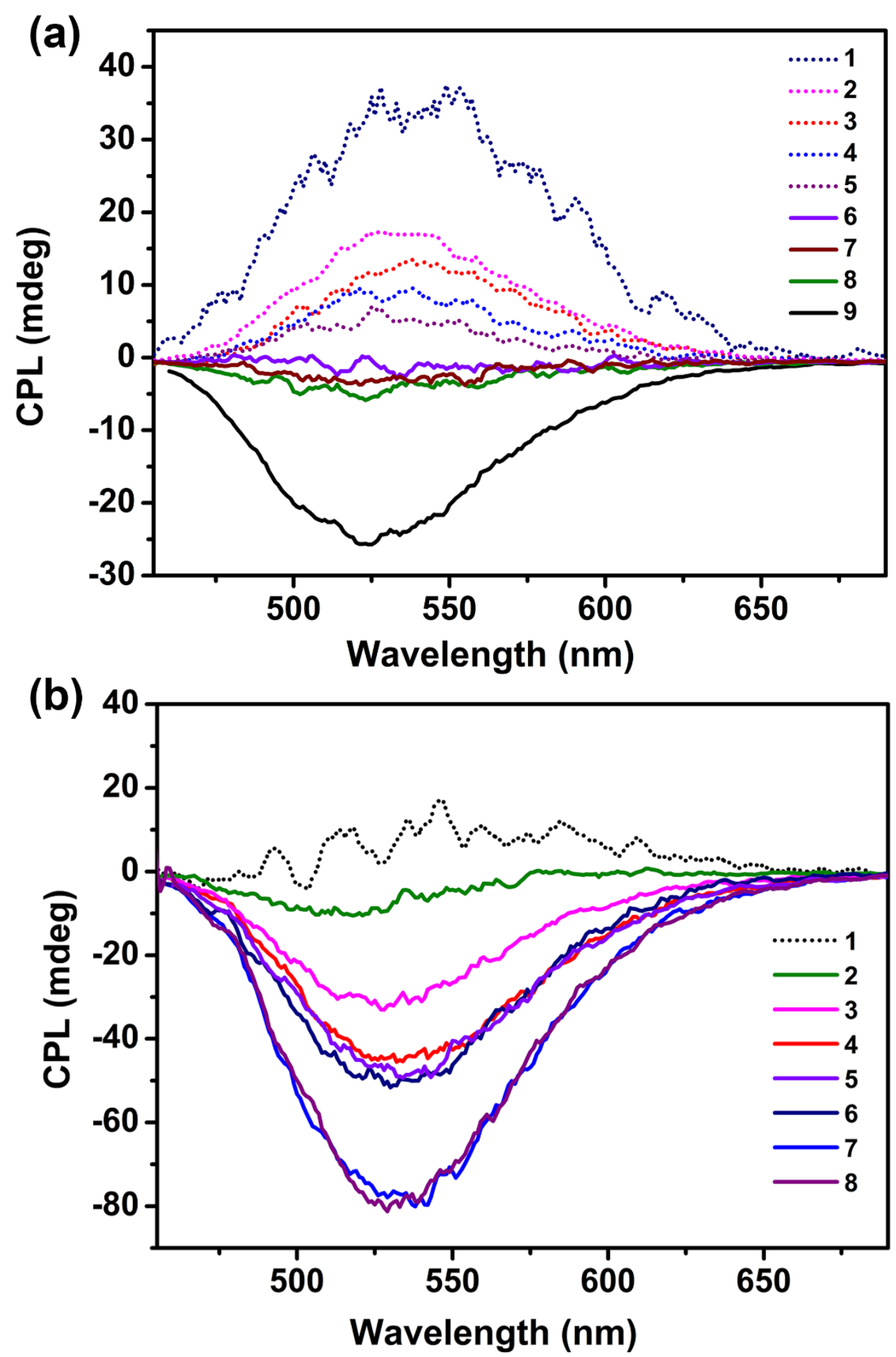

Figure S10. CPL spectra of $\left(\mathbf{K C}_{2} \mathbf{M n B r}_{4}(\mathrm{a})\right.$ and $(\mathbf{K C})_{2} \mathbf{M n C l}_{\mathbf{4}}$ (b) powder samples. The positive CPL signals are depicted by short dotted lines and the negative CPL signals are depicted by solid lines. 


\section{Supporting Tables}

Table S1. Crystal data and structure refinement for $(\mathrm{KC})_{2} \mathbf{M n C l}_{4}$ and $(\mathrm{KC})_{2} \mathrm{MnBr}_{4}$

\begin{tabular}{|c|c|c|}
\hline compound & $(\mathrm{KC})_{2} \mathrm{MnCl}_{4}$ & $(\mathrm{KC})_{2} \mathrm{MnBr}_{4}$ \\
\hline Empirical formula & $\mathrm{C}_{40} \mathrm{H}_{48} \mathrm{Cl}_{4} \mathrm{~K}_{2} \mathrm{MnO}_{12}$ & $\mathrm{C}_{40} \mathrm{H}_{48} \mathrm{Br}_{4} \mathrm{~K}_{2} \mathrm{MnO}_{12}$ \\
\hline Formula weight & 995.72 & 1173.56 \\
\hline Tempetature / K & $170 \mathrm{~K}$ & $170 \mathrm{~K}$ \\
\hline Crystal system & monoclinic & monoclinic \\
\hline Space group & $C c$ & $C c$ \\
\hline$a / \AA$ & $9.41800(10)$ & $9.5199(2)$ \\
\hline$b / \AA$ & $35.5659(3)$ & $35.8640(7)$ \\
\hline$c / \AA$ & $26.6416(2)$ & $27.0516(5)$ \\
\hline$\alpha /^{\circ}$ & 90 & 90 \\
\hline$\beta 1^{\circ}$ & $91.0080(10)$ & $90.650(2)$ \\
\hline$\gamma 1^{\circ}$ & 90 & 90 \\
\hline$V / \AA^{3}$ & $8922.48(14)$ & $9235.4(3)$ \\
\hline$Z$ & 8 & 8 \\
\hline$\rho_{\text {calc }} \mathrm{g} / \mathrm{cm}^{3}$ & 1.482 & 1.688 \\
\hline$\mu / \mathrm{mm}^{-1}$ & 6.782 & 8.478 \\
\hline $\mathrm{F}(000)$ & 4056.0 & 4696.0 \\
\hline $2 \Theta$ range for data collection $/{ }^{\circ}$ & 5.976 to 130.094 & 4.928 to 129.998 \\
\hline Reflections collected & 20464 & 47205 \\
\hline Data/restraints/parameters & $13272 / 2 / 1198$ & $12012 / 8 / 1063$ \\
\hline Goodness-of-fit on $\mathrm{F}^{2}$ & 1.067 & 1.077 \\
\hline Final $R$ indexes $[I>=2 \sigma(I)]$ & $R_{l}=0.0326, w R_{2}=0.0837$ & $R_{1}=0.0626, w R_{2}=0.1756$ \\
\hline Final $\mathrm{R}$ indexes [all data] & $R_{1}=0.0374, w R_{2}=0.0856$ & $R_{1}=0.0715, w R_{2}=0.1800$ \\
\hline Flack parameter & $-0.003(4)$ & $0.033(8)$ \\
\hline $\mathrm{CCDC}$ & 1946022 & 1946023 \\
\hline
\end{tabular}


Table S2. Selected bond distances $(\AA)$ and bond angles $\left({ }^{\circ}\right)$ for $(\mathbf{K C})_{2} \mathbf{M n C l}_{4}$

\begin{tabular}{|c|c|c|c|c|c|}
\hline \multicolumn{2}{|c|}{ Bond length $(\AA)$} & \multicolumn{4}{|c|}{ Bond angle $\left(^{\circ}\right)$} \\
\hline Mn1-Cl1 & $2.3982(12)$ & Cl3-Mn1-Cl1 & $111.95(5)$ & $\mathrm{Cl} 3-\mathrm{Mn} 1-\mathrm{Cl} 2$ & $108.13(5)$ \\
\hline $\mathrm{Mn} 1-\mathrm{Cl} 2$ & $2.3861(14)$ & Cl4-Mn1-Cl3 & $109.48(5)$ & Cl4-Mn1-Cl1 & $106.28(5)$ \\
\hline $\mathrm{Mn} 1-\mathrm{Cl} 3$ & $2.3572(13)$ & $\mathrm{Cl} 4-\mathrm{Mn} 1-\mathrm{Cl} 2$ & $114.10(5)$ & Cl2-Mn1-Cl1 & $106.93(2)$ \\
\hline $\mathrm{Mn} 1-\mathrm{Cl} 4$ & $2.3426(14)$ & $\mathrm{Cl} 6-\mathrm{Mn} 2-\mathrm{Cl} 5$ & $107.82(5)$ & Cl6-Mn2-Cl8 & $110.47(5)$ \\
\hline $\mathrm{Mn} 2-\mathrm{Cl} 5$ & $2.3955(12)$ & $\mathrm{Cl} 7-\mathrm{Mn} 2-\mathrm{Cl} 5$ & $108.71(5)$ & $\mathrm{Cl} 7-\mathrm{Mn} 2-\mathrm{Cl} 6$ & $109.12(5)$ \\
\hline $\mathrm{Mn} 2-\mathrm{Cl} 6$ & $2.3567(13)$ & Cl7-Mn2-Cl8 & $114.25(5)$ & $\mathrm{C} 18-\mathrm{Mn} 2-\mathrm{Cl} 5$ & $106.24(5)$ \\
\hline $\mathrm{Mn} 2-\mathrm{Cl} 7$ & $2.3477(13)$ & Mn1-Cl1-K1 & $138.26(5)$ & Mn1-Cl1-K2 & $117.89(5)$ \\
\hline $\mathrm{Mn} 2-\mathrm{Cl} 8$ & $2.3930(13)$ & $\mathrm{Mn} 2-\mathrm{Cl} 5-\mathrm{K} 3$ & $140.39(6)$ & $\mathrm{Mn} 2-\mathrm{Cl} 8-\mathrm{K} 4$ & $118.87(5)$ \\
\hline K1-Cl1 & $3.0979(15)$ & $\mathrm{O} 2-\mathrm{K} 1-\mathrm{Cl} 1$ & $92.98(7)$ & O5-K1-Cl1 & $95.40(7)$ \\
\hline $\mathrm{K} 2-\mathrm{Cl} 2$ & $3.0790(16)$ & O6-K1-Cl1 & $87.34(8)$ & O4-K1-Cl1 & $105.21(7)$ \\
\hline $\mathrm{K} 3-\mathrm{Cl} 5$ & $3.0604(15)$ & O1-K1-Cl1 & $85.67(8)$ & O3-K1-Cl1 & $106.55(8)$ \\
\hline \multirow[t]{9}{*}{$\mathrm{K} 4-\mathrm{Cl} 8$} & $3.0956(17)$ & O14-K3-Cl5 & $89.29(7)$ & O16-K3-Cl5 & $103.46(7)$ \\
\hline & & O18-K3-Cl5 & $90.62(8)$ & O13-K3-Cl5 & $81.90(7)$ \\
\hline & & O17-K3-Cl5 & $96.33(7)$ & O15-K3-Cl5 & $107.33(8)$ \\
\hline & & $\mathrm{O} 11-\mathrm{K} 2-\mathrm{Cl} 2$ & $106.66(8)$ & $\mathrm{O} 9-\mathrm{K} 2-\mathrm{Cl} 2$ & $92.59(8)$ \\
\hline & & $\mathrm{O} 8-\mathrm{K} 2-\mathrm{Cl} 2$ & $96.97(8)$ & $\mathrm{O} 10-\mathrm{K} 2-\mathrm{Cl} 2$ & $95.97(8)$ \\
\hline & & $\mathrm{O} 7-\mathrm{K} 2-\mathrm{Cl} 2$ & $93.98(8)$ & $\mathrm{O} 12-\mathrm{K} 2-\mathrm{Cl} 2$ & $99.28(8)$ \\
\hline & & O19-K4-Cl8 & $90.11(8)$ & $\mathrm{O} 22-\mathrm{K} 4-\mathrm{Cl} 8$ & $99.70(8)$ \\
\hline & & $\mathrm{O} 24-\mathrm{K} 4-\mathrm{Cl} 8$ & 93.82 (2) & $\mathrm{O} 21-\mathrm{K} 4-\mathrm{Cl} 8$ & $106.74(8)$ \\
\hline & & $\mathrm{O} 20-\mathrm{K} 4-\mathrm{Cl} 8$ & $94.57(7)$ & $\mathrm{O} 23-\mathrm{K} 4-\mathrm{Cl} 8$ & $96.77(8)$ \\
\hline
\end{tabular}

Symmetry operations: ${ }^{1}-1 / 2+x,-1 / 2+y,+z ;{ }^{2} 1 / 2+x, 1 / 2+y,+z$

Table S3. Selected bond distances $(\AA)$ and bond angles $\left({ }^{\circ}\right)$ for $(\mathbf{K C})_{2} \mathbf{M n B r} 4$

\begin{tabular}{ll|llll}
\hline \multicolumn{2}{c|}{ Bond length $(\AA)$} & \multicolumn{4}{|c}{ Bond angle $\left(^{\circ}\right)$} \\
\hline Mn1-Br1 & $2.531(3)$ & Br2-Mn1-Br4 & $116.25 .(12)$ & Br2-Mn1-Br1 & $104.90(11)$ \\
Mn1-Br2 & $2.528(3)$ & Br2-Mn1-Br3 & $108.21(13)$ & Br1-Mn1-Br3 & $112.41(12)$ \\
Mn1-Br3 & $2.494(3)$ & Br4-Mn1-Br3 & $108.62(11)$ & Br4-Mn1-Br1 & $106.51(12)$
\end{tabular}




\begin{tabular}{|c|c|c|c|c|c|}
\hline Mn1-Br4 & $2.480(3)$ & Br8-Mn2-Br7 & $108.50(11)$ & Br8-Mn2-Br5 & $107.71(11)$ \\
\hline Mn2-Br5 & $2.534(3)$ & Br7-Mn2-Br5 & $109.20(11)$ & Br8-Mn2-Br6 & $110.82(12)$ \\
\hline Mn2-Br6 & $2.532(3)$ & Br7-Mn2-Br6 & $115.33(12)$ & Br5-Mn2-Br6 & $104.99(11)$ \\
\hline $\mathrm{Mn} 2-\mathrm{Br} 7$ & $2.477(3)$ & $\mathrm{Mn} 1-\mathrm{Br} 2-\mathrm{K} 2$ & $112.53(12)$ & Mn1-Br1-K1 & $140.08(11)$ \\
\hline Mn2-Br8 & $2.489(3)$ & $\mathrm{Mn} 2-\mathrm{Br} 5-\mathrm{K} 3$ & $142.86(11)$ & Mn2-Br6-K4 & $114.68(11)$ \\
\hline $\mathrm{K} 1-\mathrm{Br} 1$ & $3.241(4)$ & $\mathrm{O} 1-\mathrm{K} 1-\mathrm{Br} 1$ & $83.3(3)$ & $\mathrm{O} 2-\mathrm{K} 1-\mathrm{Br} 1$ & $86.9(3)$ \\
\hline $\mathrm{K} 2-\mathrm{Br} 2$ & $3.224(4)$ & O3-K1-Br1 & $93.0(3)$ & $\mathrm{O} 4-\mathrm{K} 1-\mathrm{Br} 1$ & $100.6(3)$ \\
\hline K3-Br5 & $3.224(4)$ & O5-K1-Br1 & $101.6(3)$ & O6-K1-Br1 & $89.3(2)$ \\
\hline \multirow[t]{9}{*}{ K4-Br6 } & $3.245(5)$ & $\mathrm{O} 7-\mathrm{K} 2-\mathrm{Br} 2$ & $98.9(3)$ & $\mathrm{O} 8-\mathrm{K} 2-\mathrm{Br} 2$ & $104.6(3)$ \\
\hline & & $\mathrm{O} 9-\mathrm{K} 2-\mathrm{Br} 2$ & $92.2(3)$ & $\mathrm{O} 10-\mathrm{K} 2-\mathrm{Br} 2$ & $87.3(3)$ \\
\hline & & $\mathrm{O} 11-\mathrm{K} 2-\mathrm{Br} 2$ & 91.9 (3) & $\mathrm{O} 12-\mathrm{K} 2-\mathrm{Br} 2$ & 91.9 (3) \\
\hline & & O13-K3-Br5 & $88.7(3)$ & O14-K3-Br5 & $80.4(3)$ \\
\hline & & O15-K3-Br5 & $87.2(2)$ & O16-K3-Br5 & $102.8(3)$ \\
\hline & & O17-K3-Br5 & $98.9(3)$ & O18-K3-Br5 & $92.3(3)$ \\
\hline & & O19-K4-Br6 & $92.0(3)$ & O20-K4-Br6 & $90.2(5)$ \\
\hline & & O21-K4-Br6 & $91.5(3)$ & $\mathrm{O} 22-\mathrm{K} 4-\mathrm{Br} 6$ & $98.5(3)$ \\
\hline & & O23-K4-Br6 & $91.8(3)$ & O24-K4-Br6 & 89.7 (3) \\
\hline
\end{tabular}

Symmetry operations: ${ }^{1}-3 / 2+x, 1 / 2+y,+z ;{ }^{2}-1+x,+y,+z ;{ }^{3} 3 / 2+x,-1 / 2+y,+z ;{ }^{4} 1+x,+y,+z$

\section{Supplementary References}

(1) CrysAlisPro 2012, Agilent Technologies. Version 1.171.36.31.

(2) Sheldrick, G. M. Acta Cryst. A. 2008, 64, 112-122.

(3) Dolomanov, O. V.; Bourhis, L. J.; Gildea, R. J.; Howard, J. A. K.; Puschmann, H. J. Appl. Cryst. 2009, 42, 339-341.

(4) Sheldrick, G. M. Acta Cryst. C. 2015, 71, 3-8. 